\title{
Effects of Frequency and Jet to Surface Spacing on Synthetic Jet Impingement Heat Transfer
}

\author{
Arun Jacob, Shafi K A, K E Reby Roy
}

\begin{abstract}
Synthetic jet is a new technique for electronic chip cooling, which combines stagnant air to form a jet resulting from periodic diaphragm oscillations in a cavity. In this work, the heat transfer characteristics of a synthetic jet are investigated experimentally and numerically. A Piston-cylinder arrangement powers the synthetic jet through a circular orifice for the impingement of jet on the heated surface. Air is considered as the cooling medium. The major parameters identified to describe the impinging jet heat transfer are Reynolds number, frequency, ratio of jet spacing to diameter(Z/D) and nozzle geometry. Numerical studies have been carried out using the finite volume based commercial software ANSYS Fluent. The turbulent model used is $k-\omega$ model. The dimensionless distance between the nozzle and plate surface is in the range 2 to 16. Numerical results are in fair agreement with experimental results. As the frequency increases the average Nusselt number increases. High frequency synthetic jets were found to remove more heat than low frequency jets for small $Z / D$ ratio, while low frequency jets are more effective at larger $\mathrm{Z} / \mathrm{D}$ ratio. Nusselt number is maximum at the stagnation point and there occurs a secondary peak at lower Z/D ratios. Synthetic jet with rectangular orifice is more effective as compared to circular and square geometries.
\end{abstract}

Keywords: Frequency, Jet impingement, Nusselt Number, Synthetic Jet.

\section{INTRODUCTION}

$\mathrm{T}_{\mathrm{h}}$ he temperature control of the high dissipative components of electronic systems is a critical point in electronic cooling. Engineers have come up with several solutions such as heat sinks, forced air cooling (fans), heat pipes, which are conventional methods of cooling. However, they do have some drawbacks, such as the heat sinks need a smooth and flat surface for better heat dissipation, which costs more for manufacturing; forced air cooling (fan) needs more space in the system; heat pipes are hollow metal tubes with a thermal liquid in it, which has the potential to harm the system. To overcome these drawbacks, the synthetic jet can be potentially used for cooling applications. Impinging

Revised Manuscript Received on June 10, 2020.

* Correspondence Author

Arun Jacob*, Research Scholar, University of Kerala, Department of Mechanical Engineering, T.K.M college of Engineering, Kerala, India. E-mail: arunjacobcool@gmail.com

Shafi K A, Departmnt of Mechanical Engineering, T.K.M college of Engineering, Kerala, India. E-mail: shafika.tkm@gmail.com

Reby Roy, Department of Mechanical Engineering, T.K.M college of Engineering, Kerala, India. E-mail: rebyroy@tkmce.ac.in

(C) The Authors. Published by Blue Eyes Intelligence Engineering and Sciences Publication (BEIESP). This is an open access article under the CC BY-NC-ND license (http://creativecommons.org/licenses/by-nc-nd/4.0/) synthetic air jets can be used for heat transfer in a range of applications, which can vary from cooling a production process to thermal management of electronic systems. Synthetic jets can be produced by periodic suction and ejection of fluid from an orifice that is bounded by a cavity at the time-periodic movement of a membrane inserted into one of the cavity walls. A coherent vortex is formed and convected downstream from the jet exit, followed by a jet, during the ejection process. When the vortex flow has spread farther downstream, the ambient fluid from the vicinity of the orifice is the bulk of the high-speed air from the orifice passed away. It cancels air re-entraining into the cavity and draws the ambient air into the orifice.

Brignoni and Garimella[1], conducted studies on the effects of nozzle inlet chamfering on pressure drop and local heat transfer distribution on a small heat source. With Reynolds numbers in the turbulent system, they compared results obtained with chamfered nozzles to those obtained with a square-edged (without chamfering) nozzle of the same diameter. Due to the chamfering of the nozzle, the ratio of average heat transfer coefficient to pressure drop was increased by about 39 per cent. Anna Pavlova and Amitay [2] studied the effect of $\mathrm{Z} / \mathrm{D}$ ratio on heat transfer for different frequencies $420 \mathrm{~Hz}$ and $1200 \mathrm{~Hz}$. For frequency $420 \mathrm{~Hz}$ the improvement in Nusselt number with respect to free convection was up to $78 \%$ and optimum cooling is obtained at Z/D 6 to 18 . At frequency $1200 \mathrm{~Hz}$ the enhancement in Nusselt number with respect to free convection is up to $113 \%$ and optimum cooling is obtained at Z/D ratio 3 to 11 . High frequencies were found to remove heat better than the low frequency at a small Z/D ratio. This is due to the breakdown and mixing of vortices.

Arik et al. [3] studied the effect of Z/D ratio of steady jet and synthetic jet. At higher Z/D ratios the steady and synthetic jet heat transfer behaviour was similar up to Reynolds number 900 , above which synthetic jet showed a superior cooling performance. The Nusselt number for synthetic jet shows a weaker dependence on Reynolds number than a steady jet, especially for higher Reynolds number. Gulati and Katti [4] investigated the effects of nozzle shape, jet-to-plate spacing and Reynolds number on the local heat transfer distribution of synthetic air jet impinging on a flat surface. The Reynolds number based on hydraulic diameter ranged from 5000 to 15000 and the jet-to-plate spacing from 0.5 to 12 nozzle diameters. Up to Z/D of 6 , the Nusselt number distribution along the horizontal axis for the rectangular jet was higher in the stagnation region than those of circular and square jets. Valiorgue et al.

\section{Published By:}

Blue Eyes Intelligence Engineering

\& Sciences Publication

(C) Convriaht: All riahts reserved

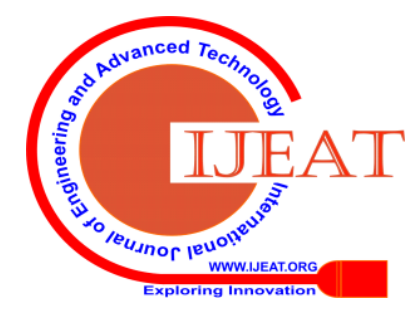




\section{Effects of Frequency and Jet to Surface Spacing on Synthetic Jet Impingement Heat Transfer}

[5] studied the effect of Z/D ratio and frequency on the heat transfer characteristics of synthetic jets. Reynolds number was in the range 1000 to 3000 . Reynolds number was directly proportional to frequency for a constant stroke length. Nusselt number increased by 33\% when Z/D ratio was increased from 2 to 6 .

Shan et al. [6] conducted experimental investigations on the effect of frequency on heat transfer of synthetic jet. The synthetic jets were produced using piston-cylinder arrangement. The frequency was in the range $8 \mathrm{~Hz}$ to $24 \mathrm{~Hz}$. The highest Nusselt number was obtained for $24 \mathrm{~Hz}$ frequency at Z/D ratio of 5.5. Fischer and Sharma [7] studied the efficacy of various turbulent models in predicting heat transfer characteristics of synthetic jet. They observed that at

$\mathrm{r} / \mathrm{D}=1.5$ the SST and SAS models give good predictions over

$k-\varepsilon$. At $\Gamma / D>2$ the $k-\varepsilon$ and $S A S$ model predicted a velocity decay. SAS Model is more suitable to the transitional flow field. Ghaffari et al. [8] performed an experimental study to investigate the cooling performance of two different synthetic jets actuated with piezoelectric actuator. Significant heat transfer degradation occurred when jets are close to the surface. A correlation was proposed for predicting Nusselt number in terms of Reynolds number, jet to-surface spacing and the stroke length.

Shaikh et al. [9] studied the effect of nozzle to plate distance for a single orifice and multi orifice synthetic jet. The maximum heat transfer coefficient with the synthetic jet was found to be 9.6 times more than that of natural convection and 3 times more than that of cooling fan used in CPU. Heat transfer in single jet was $20 \%$ more than that of a multi-jet orifice. Manoj et al. [10] numerically investigated the effect of orifice diameter and jet to plate distance. It is observed that the average heat transfer coefficient is highest for $2 \mathrm{~mm}$ diameter orifice as compared to $3 \mathrm{~mm}$ and $4 \mathrm{~mm}$ diameter orifices. For $2 \mathrm{~mm}$ diameter nozzle, maximum heat transfer occurs at a spacing of $40 \mathrm{~mm}$, and for $3 \mathrm{~mm}$ diameter nozzle maximum heat transfer occurs at $36 \mathrm{~mm}$ and for $4 \mathrm{~mm}$ diameter orifice it's about $40 \mathrm{~mm}$. As the diameter increases keeping all other parameters constant, the average heat transfer coefficient decreases. Jankee and Ganapathisubramani [11] introduced the orifice lip ratio as a new geometric parameter that can influence the performance of the jet, and studied the sensitivity of synthetic jet efficiency to this parameter. Zielinski and Solovitz [12] used particle image velocimetry (PIV) to examine the flow mechanisms in synthetic jet, using both time-averaged and phase-averaged analysis. Over the actuation cycle, phase-averaged flow fields displayed a larger, more concentrated vortex ring, which increased the maximum speed, vorticity, and circulation.

The key parameters determining the heat transfer characteristics of synthetic impinging jet are Reynolds number, frequency, the diameter of the orifice, geometry of orifice and ratio of jet spacing to jet diameter (Z/D). Experimental and numerical investigations have been carried out to see the effect of these parameters on the heat transfer characteristics.

\section{EXPERIMENTAL SETUP AND PROCEDURE}

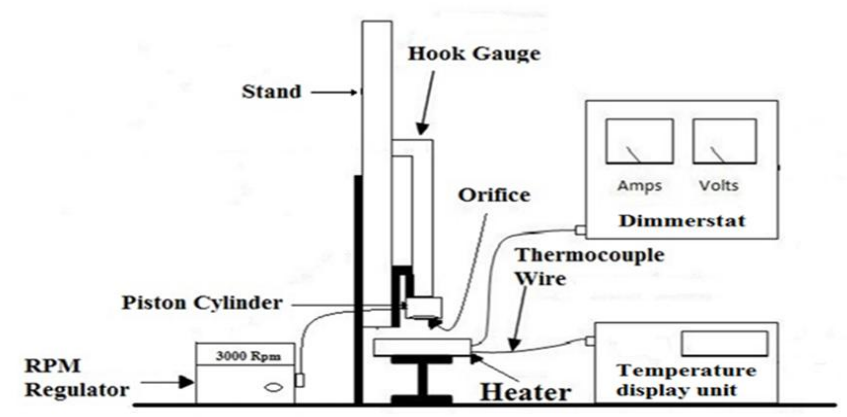

Fig. 1. The schematic diagram experimental set up

The figure 1 shows the schematic of the experimental set up The experimental setup consists of three main components: a piston cylinder assembly to produce synthetic air jet, a heated impingement surface (heater) and the instrumentation. The piston cylinder assembly is positioned in such a way that the synthetic jet impinges orthogonally on the target plate. The synthetic jet is produced by the periodic motion of a piston mounted inside a cylinder cavity. The bore of the cylinder is $15 \mathrm{~mm}$ and stroke of $26 \mathrm{~mm}$. A brushless DC motor is used for drive the piston. The frequency of the synthetic jet is varied by varying the speed of the electric motor. The thermal environment of the microprocessor is simulated using a copper plate of size $5 \mathrm{~cm}$ X $5 \mathrm{~cm}$ X $1 \mathrm{~cm}$ with a heater placed underneath (figure 3 ). Provision is there to adjust the spacing between the orifice exit and the target sur-face. The power input to the heater is varied using a dimmer-stat and the voltage and current are measured using voltmeter and ammeter, respectively. Thermocouples ( $\mathrm{T}$ type, 32 SWG) are used to measure the temperature at various locations of the copper plate. The thermocouple output is connected to a multi-channel temperature scanner. Calibrated anemometer is placed at the orifice exit to measure the jet exit velocity. The diameter of the orifice is $3 \mathrm{~mm}$.

\section{A. Experimental Procedure}

Prior to experimental measurements the thermocouples were calibrated using a constant temperature bath (Jula-boF25). The target plate is positioned in such a way that the jet strikes the top surface orthogonally. Experiments have been conducted by varying frequency, orifice diameter, nozzle to plate spacing and orifice geometry. Heat flux of $8000 \mathrm{~W} / \mathrm{m}^{2}$ i.e. corresponding to $20 \mathrm{~W}$ is supplied to the heater. The experiments are performed for the jet Reynolds number (Re) of 12000 , with a dimensionless distance between the nozzle and plate surface $(\mathrm{Z} / \mathrm{d})$ ranging from 2 to 16 . The frequency is varied in the range of ten from $10 \mathrm{~Hz}$ to $50 \mathrm{~Hz}$. The temperatures at the salient points are continuously monitored till the system reaches steady state conditions.

Published By:

Blue Eyes Intelligence Engineering

\& Sciences Publication

(C) Convriaht: All riahts reserved.

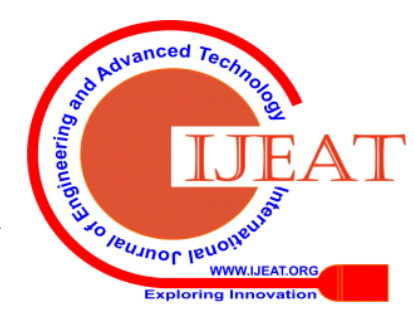




\section{NUMERICAL SETUP}

\section{A. Geometry and Boundary Conditions}

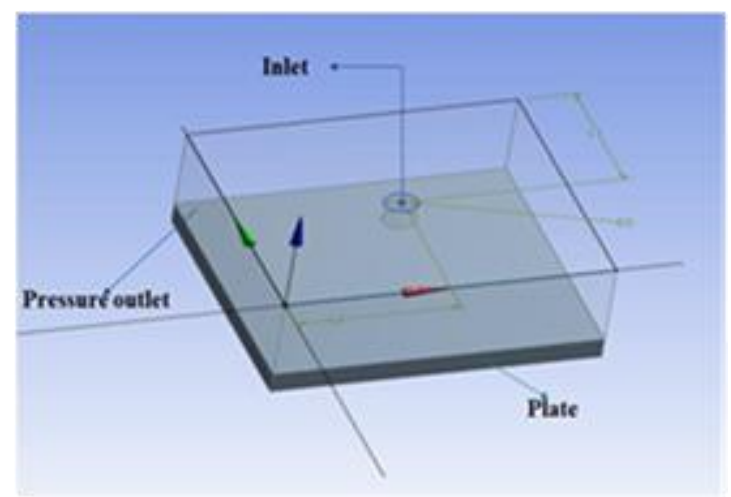

Fig. 2. The Computational domain / model

The 3D-geometry of the computational domain is modelled using ANSYS 15 Design Modeler and the same is shown in figure 2. The mesh was created using the meshing module of the ANSYS 15 workbench (figure 3). Initially, a relatively coarser mesh was used. Later a fine mesh was generated, and inflation layers are provided for all the solid-fluid wall interfaces. The turbulence model used is SST k- $\omega$ model which is found to be the best among available turbulence models for synthetic jet type flow configurations. A User Defined Function (UDF) is applied at the velocity inlet to simulate the jet and pressure outlet boundary conditions are applied the outlets. Heat flux boundary condition is applied at the bottom surface of the copper plate. Adiabatic boundary conditions are applied at other walls of the cabinet. It is assumed that the flow is continuous, incompressible and turbulent.

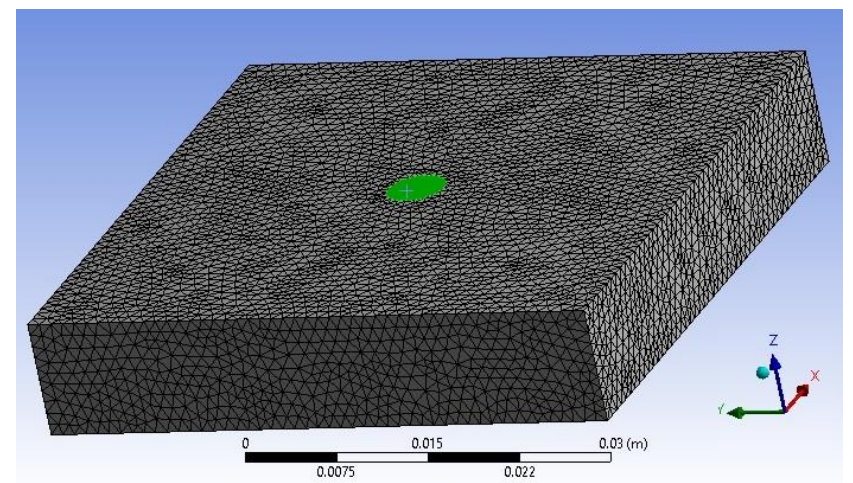

Fig. 3. The Computational domain with mesh

\section{DATA DEDUCTION}

Stroke length $(\mathrm{L})=16 \mathrm{~mm}$

Bore $(\mathrm{d})=15 \mathrm{~mm}$

Area of piston $(\mathrm{a})=\frac{\pi d^{2}}{4}$

Flow rate $(\mathrm{Q})=\mathrm{a} \mathrm{Vp}$

Where $\mathrm{Vp}$ is the velocity of the piston

Velocity of jet $(\mathrm{Vj})=\frac{Q}{A}$

A- area of jet

Reynolds number is given by
$\operatorname{Re}=\frac{\rho_{a} V j D}{\mu_{a}}$

$\rho_{a}$ - density of air

$V j$ - Velocity of jet

D - Diameter of orifice

$\mu_{a}$ - Dynamic viscosity of air

Heat transfer coefficient, $\mathrm{h}=\frac{q}{T s-T j}$

$\mathrm{q}$ - the applied heat flux,

Ts -temperature of the heated surface

$\mathrm{Tj}$ - temperature of the jet

The Nusselt number $(\mathrm{Nu})$ is widely used as the dimensionless heat transfer coefficient in the jet impingement.

Nusselt number, $\mathrm{Nu}=\frac{h D}{K a}$

Where $\mathrm{h}$ is the heat transfer coefficient

$\mathrm{D}$ is diameter of the orifice

$\mathrm{Ka}$ is the thermal conductivity of the fluid.

Where $\mathrm{q}$ is the net heat flux which is defined as

$\mathrm{q}=\frac{E I \operatorname{Cos} \phi-\text { losses }}{A p}$

Where E- applied voltage to the heater

I - current

Cos $\phi$ - power factor

Ap - Area of the target surface.

In the above equation, the losses include the conduction loss through the insulation and the radiation losses from the target surfaces. The heat loss due to conduction from the target surface to the insulating material surface is estimated separately by an experiment in which the system is brought into a steady state. The total losses are estimated as $3.31 \mathrm{~W}$. The total heat loss during the experiment is assumed to be constant. Also a small temperature difference is assumed between the inlet air jet and atmospheric.

\section{RESULTS AND DISCUSSIONS}

Experimental and numerical investigations have been carried out on synthetic jet impingement cooling for its usefulness. The heat transfer characteristics are described in terms of the Nusselt number.

\section{A. Mesh Independent Study}

A mesh sensitivity study is carried out to ascertain the accuracy of the numerical results. The mesh sensitivity study is carried out by analyzing the variation of average Nusselt number and mesh. Four different grid distributions are considered to ensure that the numerical results are grid independent, and the results obtained are shown in Fig.4. The number of elements in the various cases are 3920, 25121, 196400 , and 418200 . By comparing with the third and fourth mesh configuration in terms of stagnation Nusselt number, the variation is relatively less and it shows that $1.5 \%$.

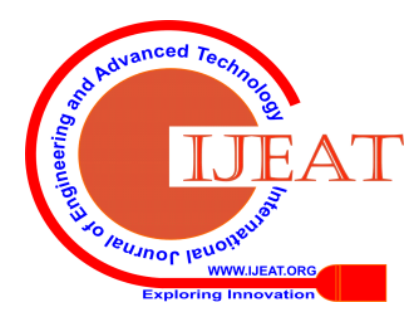




\section{Effects of Frequency and Jet to Surface Spacing on Synthetic Jet Impingement Heat Transfer}

Therefore, for further analysis, a case with 196400 elements has been adopted because it ensured a good compromise between computational time and accuracy requirements

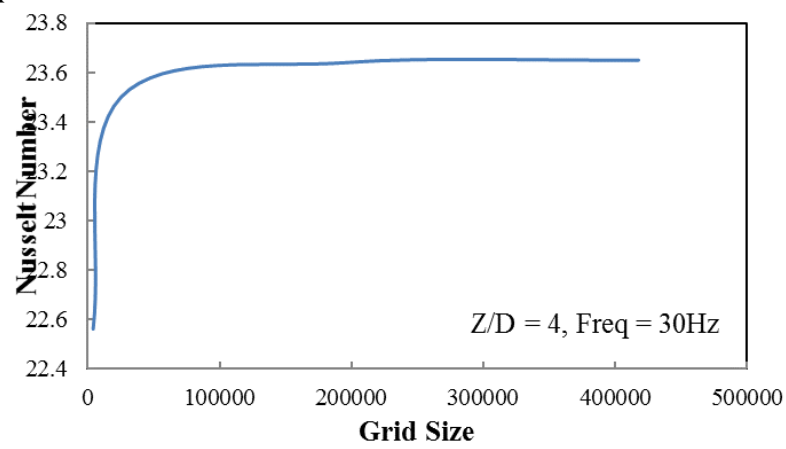

Fig. 4. Variation of average Nusselt number with number of elements

\section{B. Effects of Jet Formation Frequency and Z/D ratio on Heat Transfer}

Figure 5 shows the variation of average Nusselt number with plate to surface distance at various frequencies. Here the diameter of the orifice is $3 \mathrm{~mm}$. The results obtained from experiments and numerical studies have been shown for comparison. The Z/D ratio is varied from 2 to 16 and frequency from 10 to $50 \mathrm{~Hz}$. There is fair agreement between the values obtained from numerical and experimental studies. As the frequency increases the average Nusselt number increases. The maximum average Nusselt number increases by $40 \%$ when the frequency is increased from $10 \mathrm{~Hz}$ to $50 \mathrm{~Hz}$. At all frequencies the average Nusselt number increases with $\mathrm{Z} / \mathrm{d}$ ratio to a maximum value and then decreases. Further the optimum value of $\mathrm{Z} / \mathrm{d}$ ratio decreases with increase in frequency. For example, for $10 \mathrm{~Hz}$ the optimum Z/D ratio is 14 and that for $30 \mathrm{~Hz}$ is 10 .

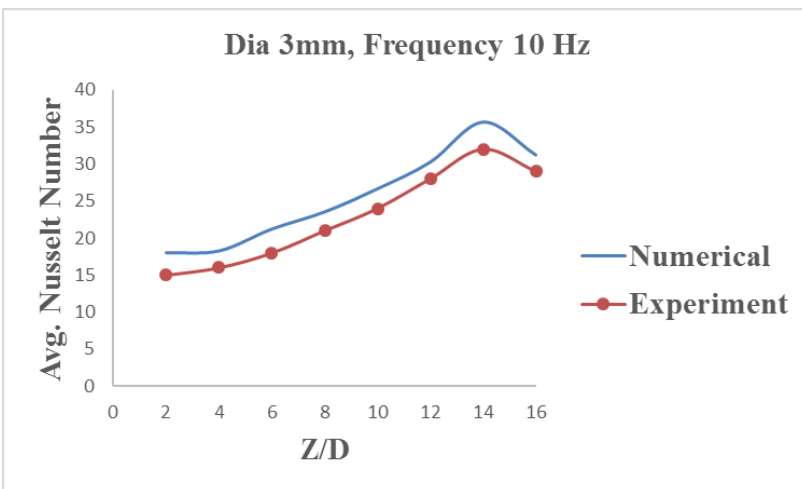

(a)

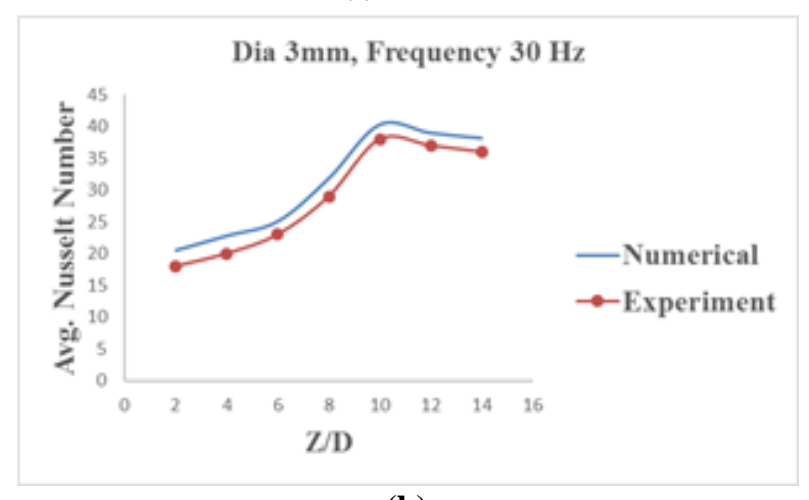

(b)

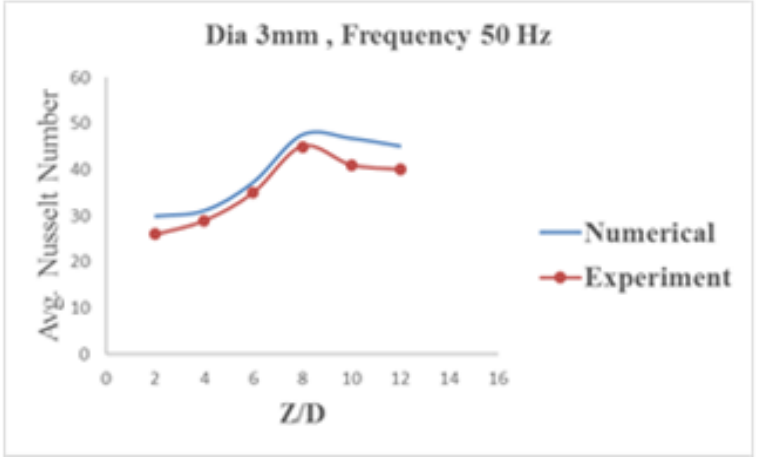

(c)

Fig. 5. Effect of ratio $\mathrm{Z} / \mathrm{D}$ ratio and frequency on average Nusselt number.(a) $10 \mathrm{~Hz}$ (b) $30 \mathrm{~Hz}$ (c) $50 \mathrm{~Hz}$. Reynolds number: 12000, Diameter of orifice: $\mathbf{3} \mathbf{~ m m}$.

High frequency synthetic jets were found to remove heat better than low frequency jets for small Z/D ratio, while low frequency jets are more effective at larger $\mathrm{Z} / \mathrm{D}$ ratio. At lower frequencies and Z/D ratios, recirculation and entrainment effects are predominant which in turn reduces the heat transfer ability.

\section{Stream wise distribution of Nusselt number}

Numerical studies were performed to study the stream wise distribution of the Nusselt number at different frequencies for a circular nozzle (figure 6). The effect of $\mathrm{Z} / \mathrm{D}$ ratio is also studied. In all cases the Nusselt number is maximum at the stagnation point and it decreases in the streamwise direction. It is observed that at a low Z/D ratio there is a secondary peak or non-uniform distribution of cooling. The secondary peak disappears as the $\mathrm{Z} / \mathrm{D}$ ratio is increased. When the distance between the jet and the plate is approximately equal to the piston's stroke duration. the synthetic jet gets enough time to develop vortices before impinging and after impingement to escape from the surface. Those vortices can split the thermal boundary layer and increase the rate of heat transfer. At higher $\mathrm{Z} / \mathrm{D}$ ratio increases the interaction and eventual collapse of the coherent vortical structures occurs leading to increased mixing and thus the heat transfer curve "spreading." If the impact of confinement is greater, there is a great chance for the recirculation of the same hot fluid that impinges on the walls of the plate and reduces the heat transferability. When the $\mathrm{Z} / \mathrm{D}$ ratio increases confinement effects are reduced, which in turn reduces the recirculation effects

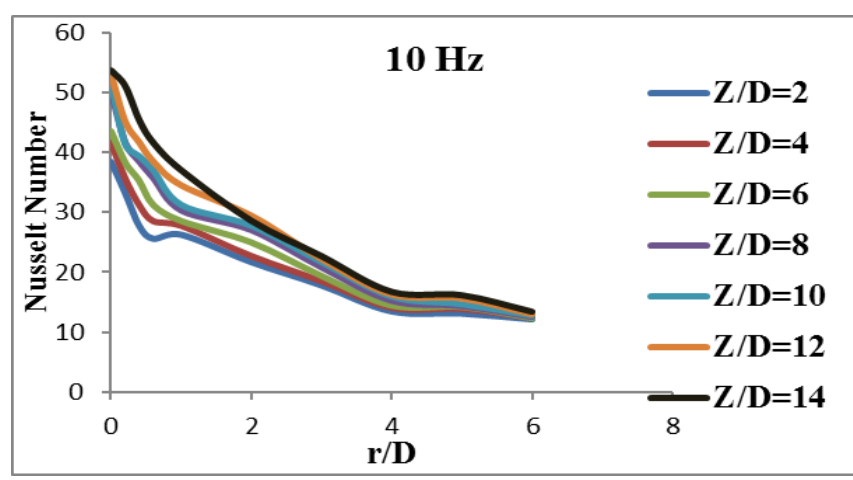

(a)

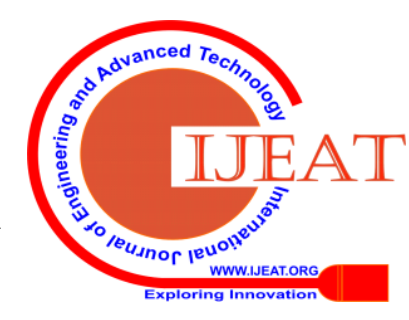




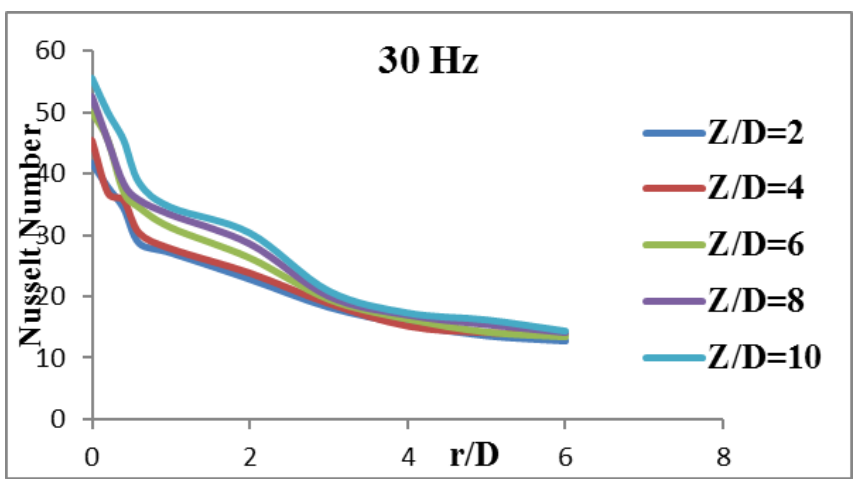

(b)

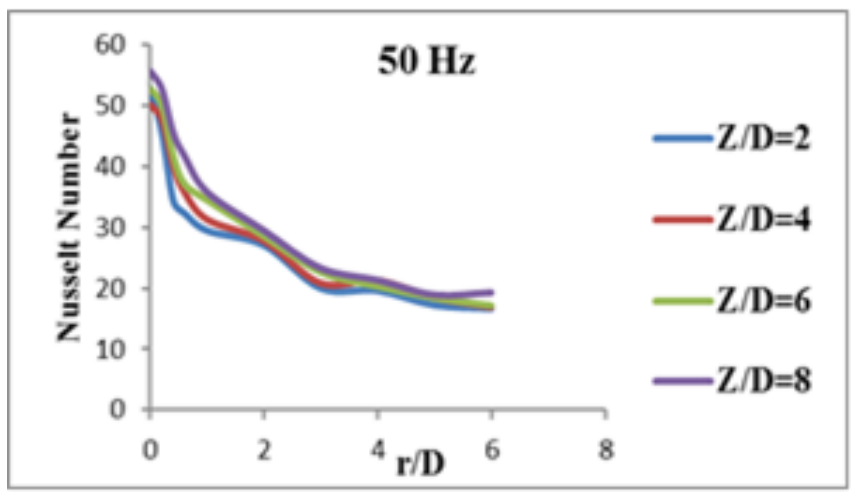

(c)

Fig. 6. Stream wise distribution of Nusselt number (a) $10 \mathrm{~Hz}$ (b) $30 \mathrm{~Hz}$ and(c) $50 \mathrm{~Hz}$

\section{Heat Transfer Characteristics of Rectangular Orifice}

The variation of local Nusselt number at the target plate at various $\mathrm{Z} / \mathrm{D}$ ratios for rectangular orifice is shown in figure 7. The impingement jet has $50 \mathrm{~Hz}$ frequency and orifice equivalent area is same as that of circular geometry. Here also the Nusselt number increases with Z/D ratio to a maximum value and then decreases. As in the case of circular geometry the optimum Z/D ratio is about 8 . However maximum Nusselt number is slightly more compared to circular case.

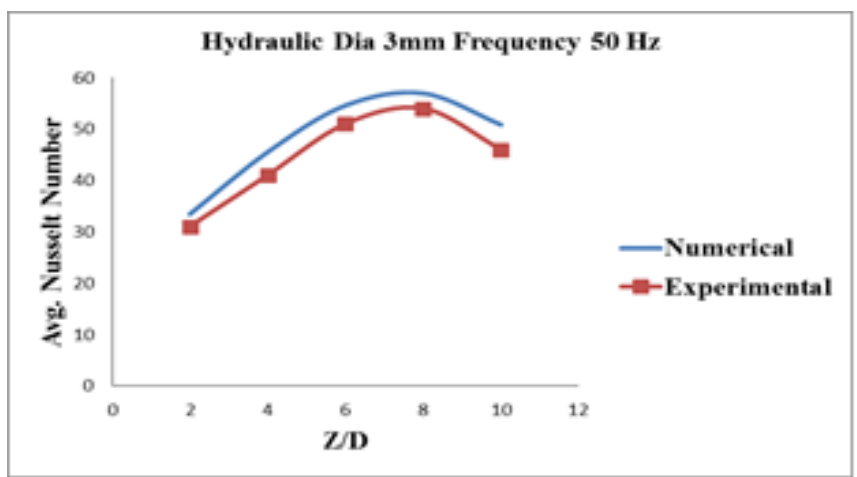

Fig. 7. Variation of average Nusselt number for rectangular nozzle

\section{E. Heat Transfer Characteristics of Square Orifice}

The variation of local Nusselt number at the target plate at various $\mathrm{Z} / \mathrm{D}$ ratios for square orifice is shown in figure 8 . The impingement jet has $50 \mathrm{~Hz}$ frequency and orifice equivalent area is same as that of circular geometry. Here also the Nusselt number increases with $\mathrm{Z} / \mathrm{D}$ ratio to a maximum value and then decreases. The optimum $\mathrm{Z} / \mathrm{D}$ ratio is about 6 . and the maximum Nusselt number is less compared to rectangular and circular configurations. Area covered by rectangular orifice is large compared to square and circular orifices, another reason more cooling is the increase in the entrainment rate for the rectangular orifice when compared to square and circular jet.

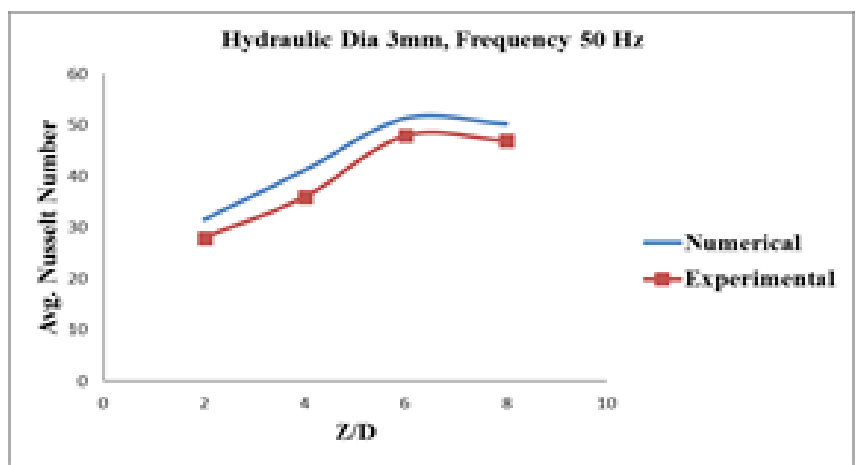

Fig. 8. Variation of Average Nusselt number for square nozzle

\section{CONCLUSION}

Experimental and numerical investigations have been carried out to study the heat transfer characteristics of synthetic jet impingement cooling system. The effect of Reynolds number, frequency, nozzle to plate distance, shape of the orifice is investigated. The major conclusions derived out of this study are (i) the numerical scheme in successful in predicting the heat transfer characteristics of synthetic jets(ii) as the frequency increases the optimum value of $\mathrm{Z} / \mathrm{D}$ ratio decreases.(iii) for the same operating conditions the optimum $\mathrm{Z} / \mathrm{D}$ ratio for synthetic jet is higher compared to steady jet(iv) high frequency synthetic jets were found to remove more heat than low frequency jets for small Z/D ratio, while low frequency jets are more effective at larger $\mathrm{Z} / \mathrm{D}$ ratio(v) Nusselt number is maximum at the stagnation point(vi) streamwise distribution of Nusselt number indicates that at lower Z/D ratios there exists a secondary peak at low Z/D ratios(vii)for the same flow area rectangular orifice has better heat transfer performance as compared to circular and square jets.

\section{REFERENCES}

1. L A Brignoni, S Garimella, "Effects of nozzle-inlet chamfering on pressure drop and heat transfer in confined air jet impingement", International Journal of Heat and Mass Transfer, April 2000, 43(7), pp. 1133-1139,

2. Anna Pavlova, Michael Amitay, "Electronic Cooling Using Synthetic Jet Impingement”, Journal of Heat Transfer. September 2006; 128(9), pp. 897-907.

3. M. Arik "Local Heat Transfer coefficients of a High-Frequency Synthetic Jet during impingement cooling over Flat Surfaces", Heat Transfer Engineering, 2008, 29(9), pp.763-773

4. P. Gulati, V. Katti, S. V. Prabhu, "Influence of the Shape of the Nozzle on Local Heat Transfer Distribution Between Smooth Flat Surface and Impinging Air Jet", International Journal of Thermal Sciences, march 2009 48(3):602-617 DOI: 10.1016/j.ijthermalsci.2008.05.002.

5. P. Valiorgue, T. Persoons, A. McGuinn, D. B. Murray, "Heat transfer mechanisms in an impinging synthetic jet for a small jet-to-surface spacing”, Experimental Thermal and Fluid Science, April 2009, 33(4), pp. 597-603

6. Shan Gao, JingZhou Zhang \& XiaoMing Tan, "Experimental study on heat transfer characteristics of synthetic jet driven by piston actuator”, Science China Technological Sciences, 2012, volume 55 , pp. 1732-1738.

Published By:

Blue Eyes Intelligence Engineering

\& Sciences Publication

(C) Convriaht: All riahts reserved

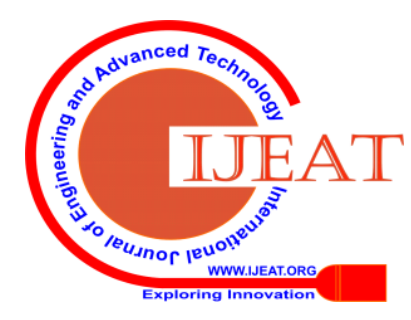




\section{Effects of Frequency and Jet to Surface Spacing on Synthetic Jet Impingement Heat Transfer}

7. C. Fischer and R. Sharma, "3D numerical simulations of a transitional axisymmetric synthetic jet", 18th Australasian Fluid Mechanics Conference Launceston, Tasmania, Australia December 2012.

8. O.Ghaffari, Mhd. Ikhlaq and M. Arik, "An Experimental Study of Impinging Synthetic Jets for Heat Transfer Augmentation", International Journal of Air-Conditioning and Refrigeration, 2015, Vol. 23, No. 3

9. Shaikh K.A., Kale S.S., Kashid A.S., "Performance evaluation of synthetic jet cooling for CPU", International Research Journal of Engineering and Technology, Jan-2016, Volume: 03 Issue: 01.

10. Manoj R, Shekhar K, Prakash T, Ganesh K, "Study of Heat transfer characteristics of Synthetic Jet", International Research Journal of Engineering and Technology, Apr -2017, Volume: 04 Issue: 04.

11. G. K. Jankee, B. Ganapathisubramani, "Influence of internal orifice geometry on synthetic jet performance", Experiments in Fluids, 2019, pp. 60:51.

12. A. J. Zielinski and S. A. Solovitz, "Flow Analysis of the Impingement of a Variable-Diameter Synthetic Jet", Journal of Flow Visualization and Image Processing, 2019, Volume:26, pp. 127-148.

\section{AUTHORS PROFILE}

Arun Jacob Research Scholar Dept. of Mechanical Engineering TKM College of Engineering Kollam, Kerala, India

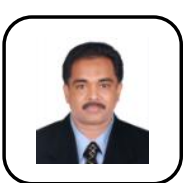

Dr. KA Shafi Professor in Mechanical Engineering, TKM College of Engineering Kollam, Kerala, India

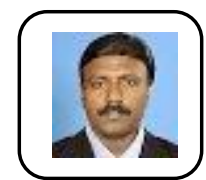

Dr KE Reby Roy Associate Professor in Mechanical Engineering TKM College of Engineering Kollam, Kerala, India.

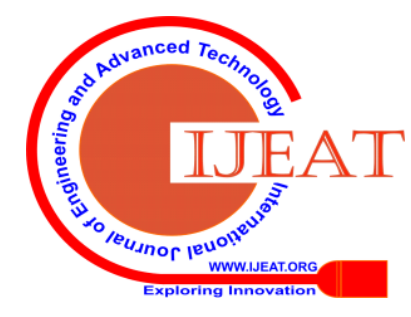

